\title{
On hybrid electroosmotic kinetics for field-effect-reconfigurable nanoparticle trapping in a four-terminal spiral microelectrode array
}

\author{
Yukun Ren, ${ }^{1,3,{ }^{*}}$ Chunlei Song, ${ }^{1}$ Weiyu Liu,,${ }^{2, *}$ Tianyi Jiang, ${ }^{1}$ Jingni Song, ${ }^{2}$ Qisheng Wu, ${ }^{2}$ Hongyuan \\ Jiang ${ }^{1, *}$
}

${ }^{1}$ State Key Laboratory of Robotics and System, Harbin Institute of Technology, West Da-zhi Street Harbin, Heilongjiang, PR China 150001

${ }^{2}$ School of Electronics and Control Engineering, and School of Highway, Chang'an University, Middle-Section of Nan'er Huan Road, Xi'an, Shaanxi, PR China 710064

${ }^{3}$ The State Key Laboratory of Nonlinear Mechanics (LNM), Institute of Mechanics, Chinese Academy of Sciences, Beijing, PR China 100190

*Corresponding Author:

Dr. Y Ren, Email: rykhit@hit.edu.cn, Fax:+86-0451-86402658, Phone: +86-0451-86418028

Dr. W Liu, Email: liuweiyu@chd.edu.cn, Fax:+86-029-82334555, Phone: +86-029-82334543

Prof. H Jiang, Email: jhy_hit@hit.edu.cn, Fax:+86-0451-86402658, Phone: +86-0451-86418028

Received: 07 29, 2018; Revised: 09 09, 2018; Accepted: 09 22, 2018

This article has been accepted for publication and undergone full peer review but has not been through the copyediting, typesetting, pagination and proofreading process, which may lead to differences between this version and the Version of Record. Please cite this article as doi: 10.1002/elps.201800325.

This article is protected by copyright. All rights reserved. 


\title{
Abbreviations
}

ACEO, ac electroosmotic; ACET, ac electrothermal; BICEO, bi-phase ICEO; DCEO, dc electroosmotic; DCEP, dc electrophoretic; DE, driving electrode; DEP, dielectrophoresis; EDL, electrical double layer; EHD, electrohydrodynamic; EO, electroosmotic; FE, floating electrode; Flow-FET, flow field-effect-transistor; FSL, flow stagnation line; GE, gate electrode; HEK, hybrid electroosmotic kinetics; ICEO, induced-charge electroosmotic; IDL, induced double layer; ITO, indium tin oxide; TWEO, traveling-wave electroosmotic

Keywords:

ac field-effect flow control; hybrid electroosmotic kinetics; induced-charge electrokinetic; microfluidics, nanoparticle concentration

Total number of words: 6000

\begin{abstract}
Induced-charge electroosmosis (ICEO) has attracted tremendous popularity for driving fluid motion from the microfluidic community since the last decade, while less attention has been paid to ICEO-based nanoparticle manipulation. We propose herein a unique concept of hybrid electroosmotic kinetics (HEK) in terms of bi-phase ICEO (BICEO) actuated in a four-terminal spiral electrode array, for effective electrokinetic enrichment of fluorescent polystyrene nanoparticles on ideally polarizable metal strips. Firstly, by alternating the applied ac voltage waves between consecutive discrete
\end{abstract}


the flow stagnation lines where the sample nanoparticles aggregate can be switched in time between two different distribution modes. Secondly, we innovatively introduce the idea of ac field-effect flow control on BICEO; by altering the combination of gating voltage sequence, not only the number of circulative particle trapping lines is doubled, but the collecting locations can be flexibly reconfigured well. Thirdly, hydrodynamic streaming of dc-biased BICEO is tested in our device design, wherein global linear electroosmosis dominates BICEO contributed from both ac and dc components, resulting in a reduction of particle enrichment area, while with a sharp increase in sample transport speed inside the bulk phase. The flow field associated with HEK is predicted using a linear asymptotic analysis under Debye-Huckel limit, with the simulation results in qualitative agreement with in-lab of nanoparticle trapping by exploiting a series of improved ICEO techniques. This work provides an affordable and field-deployable platform for real-time nanoparticle trapping in the context of dilute electrolyte.

Color online: See article online to view Figs. 1-7 in color.

Additional supporting information may be found in the online version of this article at the publisher's web-site. 


\section{Introduction}

In various biomedical and analytical applications, it is a critical step to purify or extract small particles from diverse background matrices[1]. To handle fluidic samples of low particle density, a concentration procedure is usually inevitable to confine the sample volume to an effective working range (nL to $\mu \mathrm{L}$ ) that microfluidic devices can handle[2]. For such, a number of microfluidic particle manipulation methods, such as hydrodynamics[3-5], ultrasonics[6-8], magnetism[9-11], concentration polarization[12, 13], capillary electrophoresis[14-16], electrohydrodynamics (EHD)[17-19] and dielectrophoresis (DEP)[20-23], have been studied in-depth.

Induced-charge electrokinetics takes place when an imposed background electric field $\mathbf{E}$ induces a counterionic screening layer over a polarizable surface in electrolyte, then forces that induced double-layer (IDL) into induced-charge electroosmosis (ICEO). Although the research community has fully proven the feasibility of ICEO in liquid transport and mixing over the past decade [24-26], how to make use of ICEO fluid motion to accomplish reconfigurable particle assembly has been seldom exploited. Recently, we first reported electro-convective particle trapping on the ideally polarizable surface of a central metal strip in straight microchannels[27-31]. Since particle preconcentration by lateral ICEO vortex flow arises from indirect fluidic drag force, ICEO trapping has a greater actuating range than nonlinear electrokinetic force acting directly on particle themselves[32], mainly including conventional DEP attraction/repulsion by a field gradient and traveling-wave DEP pumping along a progressively phase-shifted electrode track[33].

Although a series of ICEO particle manipulation approach has been reported, there is still a need to implement targeted enrichment of colloidal particles on a relatively broad scale. In view of this, we propose herein an unique physical concept of hybrid electroosmotic kinetics (HEK), in terms of bi-phase ICEO (BICEO) with two applied ac voltage signals of $180^{\circ}$ phase difference for switching the sample collection state in time at our wishes, as well as a more advanced version, termed as 'ac field-effect flow control on BICEO', in analogy to flow field-effect-transistor (flow-FET)[34-36] in 
microfabricated fluidic networks for realizing rectifications in dc electroosmotic (DCEO) pump flow. We make use of a spiral electrode array to construct the ideally polarizable interface for generating fast ICEO fluid motion in its vicinity, wherein four isolated electrode strips are in direct contact with electrolyte solution, so merely several volts are enough to actuate electrically the microfluidic device for position-controllable particle concentration adjacent to the metal surface; this forms a stark contrast with the technique of flow-FET developed most initially for DCEO, in which a relatively thick dielectric membrane incorporated in the three-capacitor model results in the requirement of very high gate voltages (usually $50 \mathrm{~V}-1000 \mathrm{~V}$ ), so as to accelerate appreciably the pump flow rate. In addition, the feasibility of dc-biased BICEO in causing particle trapping is tested experimentally, and the time need for particle trapping to reach a steady state is reduced to great extent due to the contribution from three distinct components of electroosmosis. These results support that HEK has great potential to enable flexible and large-scale nanoparticle concentrators in the context of microfluidics.

\section{Materials and method}

\subsection{Experimental}

To demonstrate the feasibility of current HEK theory in flexible sample manipulation, a multiphase electrokinetic microdevice is fabricated and then we carry out the particle trapping experiment. Multilayer fabrication process has to be employed if the configuration of a linear electrode array of different voltage phases is adopted. To avoid complicated wiring, we elaborate a special device design of a confocal spiral four-terminal electrode array of $\mathrm{N}=5$ repeating wavelength, as shown in Fig.1(a). These conducting rings are deposited on an insulating glass substrate, and covered by a microchannel with two opening cylindrical electrolytic ports of $1.5 \mathrm{~mm}$ in radius. The four circulating metal strips share an equal width of $\mathrm{L}_{\mathrm{E}}=100 \mu \mathrm{m}$, and the nearest gap size between adjacent electrode phases equals $\mathrm{L}_{\mathrm{G}}=30 \mu \mathrm{m}$. Consequently, the wavelength of single repeating spatial period is $\mathrm{L}=4\left(\mathrm{~L}_{\mathrm{E}}+\mathrm{L}_{\mathrm{G}}\right)=520 \mu \mathrm{m}$, the diameter of the whole helix is about $6 \mathrm{~mm}$. One great benefit of this circulating design is that, 
inter-phase insulation and external wiring of the four sequential terminals can be accomplished at the periphery of the circular electrode system at a time, with much more convenience of operation compared to that for a linear array geometry[37].

The fabrication process of the chip used in current experiment is based on the classical soft-lithography technique. Thin-film indium tin oxide (ITO) of high degree of transparency is employed to forge the electrode structure. First, the annular four-terminal metal strip array is by wet etching the ITO material. Subsequently, a PDMS microfluidic channel with $500 \mu \mathrm{m}$ in height is polymerized by using a laser-ablated PMMA pattern as the template. Eventually, PDMS chamber and glass substrate with the circulative electrode pattern are aligned under an optical microscope and bonded to form an intact microdevice through oxygen plasma treatment. The microfluidic particle concentrator (Fig.1(a)) makes use of BICEO to concentrate nanoparticle samples, with the core structure of four-terminal ITO electrode array demonstrated in Fig.1(b).

We choose aqueous saline solution as an eligible working fluid of moderate dynamic viscosity, the ionic conductivity of which is controlled by concocting $\mathrm{KCl}$ electrolyte and monitored via a conductivity analyzer. The solution conductivity for preconcentration experiment is $\sigma=0.001 \mathrm{~S} / \mathrm{m}$, which is appropriate for actuating ICEO in the absence of steric effect [38-40]. It is noteworthy that, met the thin layer approximation as well, with $\lambda_{D} / 0.5 \mathrm{~L}=0.00075 \ll$. Considering the low ionic strength of the bulk fluid, the ITO metal strips can be recognized as ideally polarizable solid in this situation. The particles used in our study were polystyrene nanospheres (Molecular Probes Co) with radius of $r=250 \mathrm{~nm}$ emitting green fluorescence. To prepare fluorescent polystyrene a $20 \mu \mathrm{L}$ suspending solution containing $10 \%$ particles was diluted with the $10 \mu \mathrm{S} / \mathrm{cm} \mathrm{KCl}$ solution into $\mathrm{mL}$, so as to keep track of the dynamic trapping process of latex beads due to electroosmotic The liquid suspension with manipulatable nanoparticles is then injected into the fluidic chamber and finally fills it. 
We make use of a commercial multichannel function generator (TGA12104, TTi, UK) to generate low-frequency ac voltage signals with arbitrary phase relation. In practical experiments, there are some optional electric field conditions, including sinusoidal signals of $180^{\circ}$ phase difference, dc-biased ac voltages are imposed to some of the four terminals; otherwise, the metal strips free from external wiring (wireless bipolar electrode) float in potential. Waveform of the applied voltage is monitored using a commercial digital oscilloscope (TDS2024, Tektronix, USA).

\subsection{Theory and mathematical model of HEK}

In ICEO, the normal field lines are responsible for charging the capacitive IDL, which is then driven the tangential field component of a same electrical source into electroconvection. For this reason, vortex streaming has a second-order voltage dependence, and thereby survives in low-frequency ac fields. In the scientific history, following the pioneering work of Ramos and co-workers on ACEO one coplanar microelectrode pair, Bazant and Squires[41, 42] pointed out that an applied background electric field can trigger its own induced free charge within the induced double layer (IDL) adjacent to a polarizable solid/electrolyte interface, which exerts a nonlinear Coulomb force inside the thin boundary layer; so, they coin the term 'induced-charge electroosmosis (ICEO)' to describe the resulted physical phenomenon of nonlinear electroosmotic streaming on conducting or even weakly polarizable surfaces. ICEO conceptually encompasses ACEO[43]/TWEO[44, 45](traveling-wave electroosmosis) on a driving electrode array, and ICEO on bipolar metal[46-49]/semiconductor[50-52]/leaky dielectric[53] solid surfaces, as well as electroosmosis of second kind in the extended space charge layer near a perm-selective membrane[54]. This kind of EHD brings about great benefits in reducing Faradaic reactions and suppressing bubble generations, as well as engendering faster electroconvection than traditional linear electroosmosis in dc fields, taking into account the nonlinear character of ICEO fluid motion[55].

In current analysis, depending on the specific experimental condition, ACEO, ac-ICEO (ICEO caused by ac field component), dc-ICEO (ICEO due to a dc voltage offset), and conventional DCEO 
may coexist along the spiral trail; so, it is actually an insurmountable task to obtain explicit analytical solution of electroosmotic flow field for this full 3-D geometry (Fig.1(a)) beyond dc limit, where electrochemical ion relaxation leads to incomplete Debye screening.

In addition, the case of a dc bias has in effect two Fourier modes, $f_{1}=0$ and $f_{2}=\omega / 2 \pi$. It involves multifrequency electroosmotic streaming, and the dc and ac fields can be separately resolved according to the superposition principle in nonlinear electrokinetics, in analogy to the concept of multifrequency DEP[56]. We carry out numerical simulation to calculate the coordinate-dependent electrostatic potential and various kinds of EO flow field, using a commercial software package, COMSOL Multiphysics (version 5.3a). The ac (using phasor amplitude denoted by a tilde) and dc electric field components in the bulk of the suspension medium are dictated by Eq.(1a) and Eq.(1b), respectively, with the boundary conditions on different surfaces explicitly presented in Table 1. Once the dc and ac potential distributions are known, we can predict the time-averaged ac-ICEO electrokinetic slip flow on the four electrode terminals, the steady dc-ICEO slipping on the bipolar metal strips, and traditional DCEO adjacent to channel walls via Eq.(5), (4b) and (4c), respectively.

The theoretical analysis takes advantage of the linear RC circuit theory in terms of electrode polarization under the asymptotic limit of small Dukhin number and ultrathin electric double layer (EDL). Speak of electrochemical transport of ionic species in aqueous solution, the ratio of diffusive to conductive mass transfer is $\mathrm{D} \nabla \rho_{\text {free }} / \sigma E_{n}^{E D L}=\left(\lambda_{\mathrm{D}} / \mathrm{h}\right)^{2}$ [57-59] (see Nomenclature), indicating the diffuse screening charge, either induced or fixed, is merely significant within the Debye layer. Accordingly, the net conduction current $\boldsymbol{n} \cdot\left(\sigma \boldsymbol{E}-\mathrm{D} \nabla \rho_{\text {free }}\right)$ vanishes along the normal direction of electrode surface. With a higher field frequency, however, displacement current flows across the EDL, which would make charging dynamics incomplete and out-of-phase polarization noticeable.

For mathematical simulation, we can divide the entire fluidic system into two interconnected regions, including the EDL at electrode/electrolyte interface and bulk fluid. Within the latter, the 
space charge density vanishes considering electroneutrality, so that the ac and dc electrostatic potential are determined by Laplace equations Eq.(1a) and Eq.(1b), respectively, according to current conservation:

$$
\begin{aligned}
& \nabla^{2} \phi_{\mathrm{ac}}=0 \quad \text { (for ac field component) } \\
& \nabla^{2} \phi_{\mathrm{dc}}=0 \quad \text { (for dc field component) }
\end{aligned}
$$

Under Debye-Huckel limit, we disregard those effects in non-ideal conditions, including Faradaic current injection, ion overcrowding and non-uniform surface electrokinetic transport etc. The EDL adjacent to the structural interfaces is comprised of a compact layer of capacitance $\mathrm{C}_{\mathrm{S}}$, and a diffuse layer of capacitance $C_{D}=\varepsilon / \lambda_{D}$, which are in serial connection to support the entire overpotential at the electrode surface. Here, $\lambda_{D}$ denotes the thickness of EDL, and $\varepsilon$ the solution permittivity. The linear EDL capacity is the combination of the above two layers, i.e. $C_{0}=C_{D} C_{S} /\left(C_{D}+C_{S}\right)=C_{D} /(1+\delta)$, in terms of the surface capacitance ratio $\delta=C_{D} / C_{s}$. Since the electrode surface is impenetrable to ions, current continuity condition for ac and dc fields in the bulk right outside the EDL is given by Eq.(2a) and Eq.(2b,2c), respectively:

$$
\begin{gathered}
\sigma \boldsymbol{n} \cdot \nabla \phi_{\mathrm{ac}}=j \omega \frac{C_{\mathrm{D}}}{1+\delta}\left(\phi_{\mathrm{ac}}-\phi_{\mathrm{aci}}\right) \text { for the } i \text { th terminal in ac field (2a) } \\
\sigma \boldsymbol{n} \cdot \nabla \phi_{d c}=0, \phi_{d c \mathrm{c}}=0.5 \mathrm{~V} \quad \text { for } i=2 \text { and } 4 \text { in dc field (2b) } \\
\phi_{d c 1}=1 \mathrm{~V}, \phi_{\mathrm{dc} 3}=0 \mathrm{~V} \text { for } i=1 \text { and } 3 \text { respectively in dc field (2c) }
\end{gathered}
$$

Where $\sigma$ is the liquid conductivity, $\omega=2 \pi f$ the angular electric field frequency, $f$ the linear frequency, tilde ' $\sim$ ' the phasor amplitude of ac field variables, $\phi_{\mathrm{ac}} / \phi_{\mathrm{dc}}$ the ac/dc potential in the bulk right outside the EDL. The subscripts ac and dc represent the ac and dc field component, and $i$ the $i$ th strip terminal (See Nomenclature). $\phi_{\text {aci }}$ denotes the ac body potential of $i$ th terminal, $\phi_{d c i}$ the dc 
counterpart, and $\boldsymbol{n}$ the unit normal on the metal electrodes. In Eq.(2), any electrode reaction is excluded for linear asymptotic analysis, namely, the EDL can merely be short circuited by displacement current in a time-dependent electric field. Effect of counterionic screening is reflected by imposing Eq.(2a) and (2b) at the liquid/electrode interface. Nondimensionalization of Eq.(2a) gives rise to a characteristic double-layer relaxation frequency $f_{R C}=(1+\delta) \sigma \lambda_{D} / 2 \pi \varepsilon R=\mathrm{O}(100) \mathrm{Hz}$ in the context of dilute electrolyte.

A part of the entire double-layer voltage drops across the diffuse layer, giving rise to a harmonic inducing zeta potential $\zeta_{\text {aci }}$, a static zeta potential $\zeta_{\text {dci }}$, and the fixed counterpart $\zeta_{\text {fixed }}$ on channel sidewalls of negative surface charge density $\sigma_{\text {free }}$ to actuate EO streaming on the spiral array:

$$
\begin{gathered}
\zeta_{\mathrm{aci}}=\frac{1}{1+\delta}\left(\phi_{\mathrm{aci}}-\phi_{\mathrm{ac}}\right) \quad(\text { zeta potential phasor for ac field) (3a) } \\
\zeta_{\mathrm{dci}}=\frac{1}{1+\delta}\left(\phi_{\mathrm{dci}}-\phi_{\mathrm{dc}}\right) \quad(\text { zeta potential for dc field) }(3 \mathrm{~b}) \\
\zeta_{\text {fixed }}=\frac{\sigma_{\text {free }} \lambda_{D}}{\varepsilon} \quad(\text { fixed zeta potential) }(3 \mathrm{c})
\end{gathered}
$$

Since the flat annular structure is adhered to the glass base in practice, Coulomb force within the Debye layer dominates any other electrokinetic phenomenon, resulting in three types of EO slipping at the sharp material interface:

$$
\begin{gathered}
\boldsymbol{u}_{s}^{a c I C E O}=\frac{\varepsilon}{\eta(1+\delta)} \operatorname{Re}\left(\left(\phi_{a c}-\tilde{\zeta}, \quad, \quad \mathrm{e}\left(\left(\boldsymbol{E}_{a c}-\boldsymbol{E}_{a c} \cdot \boldsymbol{n} \cdot \boldsymbol{n}\right) e^{j \omega t}\right)\right.\right. \\
\boldsymbol{u}_{s}^{\mathrm{dcCEOO}}=\frac{\varepsilon}{\eta(1+\delta)}\left(\phi_{d c}-\phi_{d c i}\right)\left(\boldsymbol{E}_{d c}-\boldsymbol{E}_{d c} \cdot \boldsymbol{n} \cdot \boldsymbol{n}\right) \quad(4 \mathrm{~b}) \\
\boldsymbol{u}_{s}^{D C E O}=-\frac{\varepsilon \zeta_{\text {fixed }}}{\eta(1+\delta)}\left(\boldsymbol{E}_{d c}-\boldsymbol{E}_{d c} \cdot \boldsymbol{n} \cdot \boldsymbol{n}\right) \quad(4 \mathrm{c})
\end{gathered}
$$


For analytical convenience, we use the time-averaged counterpart of Eq.(4a):

$$
\left\langle\boldsymbol{u}_{s}^{a c I C E O}\right\rangle=\frac{\varepsilon}{2 \eta(1+\delta)} \operatorname{Re}\left(\left(\phi_{a c}-\tilde{\varsigma}, \quad \boldsymbol{E}-\boldsymbol{E}_{a c} \cdot \boldsymbol{n} \cdot \boldsymbol{n}\right)^{*}\right)
$$

Where the asterisk ${ }^{*}$ is the complex conjugate, and $<>$ the time-average operator. In this way, the steady component of the slip velocity due to HEK is the sum of Eq.(5), Eq.(4b) and Eq.(4c):

$$
\left\langle\boldsymbol{u}_{\mathrm{S}}\right\rangle=\left\langle\boldsymbol{u}_{s}^{a c I C E O}\right\rangle+\boldsymbol{u}_{s}^{\mathrm{dcICEO}}+\boldsymbol{u}_{s}^{D C E O}
$$

Since the Reynolds number is sufficiently small (see Nomenclature), the linear superposition principle holds true in our microdevice. In this case, for the steady-state and incompressible Newtonian fluid, the velocity field fulfills the Stokes equation, which is subjected to the synthetic EO slipping $\left\langle\boldsymbol{u}_{\mathrm{S}}\right\rangle$ on polarizable surface:

$$
\begin{gathered}
-\nabla p+\eta \nabla^{2} \boldsymbol{u}=0 \quad(\text { momentum conservation) (7a) } \\
\nabla \cdot \boldsymbol{u}=0 \quad(\text { mass conservation) }(7 \mathrm{~b})
\end{gathered}
$$

Where $\eta$ denotes the liquid dynamic viscosity, and $p$ the hydraulic pressure.

\subsection{Numerical simulation}

We solve the Laplace equations Eq.(1a) and Eq.(1b) for the ac potential phasor and dc potential using two set of electric current $(\mathrm{ec})$ modules inherently included in Comsol Multiphysics. With the $1^{\text {st }}$ one, the ac field Eq.(1a) is subjected to IDL capacitive charging on the four conducting terminals Eq.(2a), and insulating condition on other surfaces. With the $2^{\text {nd }}$ ec module, dc field Eq.(1b) is subjected to complete charging of IDL on the $2^{\text {nd }}$ and $4^{\text {th }}$ terminal of identical floating potential $\phi=0.5 \mathrm{~V}$, and given voltage penetration of $\phi=1 \mathrm{~V}$ adjacent to the $1^{\text {st }}$ and $3^{\text {rd }}$ terminal with a dc bias. 
Once both the ac and dc field components are known, they are inserted into Eq.(6) to acquire the time-averaged EO slipping flow. Subsequently, the HEK vortex flow field in the bulk Eq.(7) is computed by imparting Eq.(6) to the polarizable interfaces. The 3D computational domain is similar to Fig. 1, with the PDMS chamber of $\mathrm{H}=500 \mu \mathrm{m}$ in physical height. Free tetrahedral meshes are hired in the numerical simulation, and the maximum grid dimension in the vicinity of electrode edges is designated to be no more than one-tenth $(10 \mu \mathrm{m})$ of the width of an individual metal strip, in terms of a growth factor of 1.1 as the meshes spread from the electrode surface to the bulk suspension. The distribution of meshes cannot be further refined, since doing this would exceed the limit of our available computer resource (Intel i7-7700K, 64G DDR4 3000MHz, ROG Z270). Though we are not able to test grid-independence because of this restriction, the simulation results can still capture the basic feature of HEK flow field manifesting as a series of micro-vortices above the electrode array. During the numerical calculation, we prefer to utilize the PARDISO solver considering its quicker iteration speed. The Reynolds number here is no more than a unit, i.e., $R e y=\rho_{\mathrm{f}} \mathrm{u} R / \eta \sim \mathrm{O}(0.1)<1$ for $\mathrm{u}=1 \mathrm{~mm} / \mathrm{s}$ and $R=100 \mu \mathrm{m}$, so that HEK slipping flow is laminar, not turbulent in practice. Even so, under AC harmonic forcing, BICEO slip can still give rise to time-averaged vortex flow field in the bulk, which push the latex beads onto the annular electrode array from the liquid suspension, by taking its second-order voltage-dependence into account.

\section{Results and discussion}

\subsection{Particle trapping via BICEO}

We first test the feasibility of standard BICEO in nanoparticle enrichment over the circular electrode array. Irrespective of multifrequency triggering, BICEO means double ac voltage signals of same frequency but opposite phases are used to actuate nonlinear EO streaming (Fig.2(a)). In this sense, we consider two conditions of electrical connection for BICEO. The first scheme (state 1) is to apply $\phi_{a c 1}(\mathrm{t})=\mathrm{A}_{1} \cos (\omega \mathrm{t})$ to the $1^{\text {st }}$ terminal and $\phi_{a c 3}(\mathrm{t})=\mathrm{A}_{3} \cos \left(\omega \mathrm{t}+180^{\circ}\right)$ to the $3^{\text {rd }}$ terminal, while the $2^{\text {nd }}$ and $4^{\text {th }}$ terminal float in the harmonic field. The second choice (state 2 ) is to 
impose $\phi_{a c 2}(\mathrm{t})=\mathrm{A}_{2} \cos (\omega \mathrm{t})$ to the $2^{\text {nd }}$ terminal and $\quad \phi_{a c 4}(\mathrm{t})=\mathrm{A}_{4} \cos \left(\omega \mathrm{t}+180^{\circ}\right)$ to the $4^{\text {th }}$ terminal, with the $1^{\text {st }}$ and $3^{\text {rd }}$ one free from external powering. In practice, the voltage amplitude $A_{1}=A_{2}=A_{3}=A_{4}=2 V$ is sufficient to capture nanoparticles without damaging the electrodes.

In this way, the two experimental approaches generate a voltage difference of $4 \mathrm{~V}$ across the $1^{\text {st }}$ and $3^{\text {rd }}$ terminal (Fig.2(b)), and the $2^{\text {nd }}$ and $4^{\text {th }}$ terminal, respectively. Since the interfacial capacitor is incessantly charged and discharged by the conduction current from the resistor of the fluid bulk, both ACEO slip flow on driving electrodes (DE) and ICEO slipping on floating electrodes (FE) have a strong dependence on the imposed field frequency $f$, as shown in Fig.2(e). In DC limit below reciprocal RC time constant $f_{R C}=(1+\delta) \sigma \lambda_{D} / 2 \pi \varepsilon R$, most of the applied voltage drops across the diffuse screening cloud, that is, the occurrence of complete Debye screening, resulting in no tangential field right outside the EDL that forces the induced charge cloud into negligible small electroconvection. With field frequency much higher than inverse RC time scale, since the voltage polarity changes too fast, there is not enough time for the ionic charge to accumulate on the ideally polarizable surface within each half cycle, that is, incomplete Debye screening takes place, and all the electrode terminals recover to their original role of ideal conductors, which makes electrokinetic slipping vanish once again (Fig.2(e), $\mathrm{f}=10 \mathrm{kHz}$ ). Consequently, BICEO vortex streaming becomes merely observable in an intermediate frequency range around $f_{R C}$, which is on the order of $\mathrm{O}(100) \mathrm{Hz}$.

To realize electroconvective particle trapping, however, the upward ICEO fluidic drag force cannot be excessively large, or else it would overcome the downward sedimentation and break the stress balance on latex beads, giving rise to unstable particle rotating motion above the annular electrode array. Accordingly, a suitable field frequency $f=50 \mathrm{~Hz}$ is chosen in priority in BICEO concentrating experiments. Under this circumstance, the floating electrodes exempt from external energizing behave as perfect insulators beyond a distance scale of $\mathrm{O}\left(\lambda_{\mathrm{D}}\right)$ due to the block of ionic current on ideally polarizable surfaces, as indicated by the parallel electric field lines above FE in Fig. 2(b). A lower 
induced polarizability of IDL at FE/electrolyte interface under 50Hz makes ICEO flow velocity $(\sim 100 \mu \mathrm{m} / \mathrm{s})$ weaker than ACEO streaming on driving electrodes $(\sim 200 \mu \mathrm{m} / \mathrm{s})$. In light of this, nanoparticles are supposed to collect selectively on unipolar DE with equidistance rather than bipolar FE, as schematically shown in Fig.2(c) and Fig.2(d) for status 1 and 2, respectively.

At the steady state of each experimental condition, flow stagnation lines (FSL) responsible for particle trapping would generate selectively on a half of the twenty sequential circles. This reminds us to change the voltage supply between these two working states, with the specific assembly pattern shown in Fig.3(a) and Fig.3(c), so that the circulatory FSL where latex beads aggregate can be switched in time at our wishes (see Supporting Movie 1 for in-lab observation of a back and forth switching of equidistant particle assembly lines driven by BICEO). Interestingly, as shown in Fig.3(b), there is a self-assembly transition state between the two operating states, wherein nanoparticles are gradually driven by BICEO electroconvection to move from current ten electrode bars to the other ten counterparts, and at an intermediate time point, it looks like particle trapping lines are developed above all the twenty annular strips. This situation is erratic, however, and is then quickly converted to status 1 (Fig.3(a)) or 2 (Fig.3(c)), depending on specific switching sequence of imposed ac voltage waves. In addition, BICEO not only produces a series of micro-vortices on electrode surface (Fig.2(c)(d)), but induces a steady pump flow component as well, as exhibited in Fig.3(d), which is acquired by superimposing a series of consecutive image frames included in experimental video. This net pump component of BICEO is originated by a subtle change of signal phase along the annular electrode track, and constantly transports colloidal samples in the outward direction, resulting in an enhancement of the horizontal flow flux and thereby concentrating performance of latex nanospheres.

\subsection{Reconfigurable self-assembly using ac field-effect flow control on BICEO}

On the basis of previous study on controllable particle trapping by periodic BICEO eddies, we then introduce the approach of flow-FET to cause symmetry breaking in BICEO flow field, i.e. ac 
field-effect flow control on BICEO. Specifically, in addition to the biphase voltage waves $A \cos (\omega t)$ and $A \cos \left(\omega \mathrm{t}+180^{\circ}\right)$ applied to the two driving terminals $\left(1^{\text {st }}\right.$ and $\left.3^{\text {rd }}\right)$, the other two terminals $\left(2^{\text {nd }}\right.$ and $4^{\text {th }}$ ) are no longer floating, but serve as gate electrodes (GE) for the sake of establishing an ac flow field-effect transistor (ac-flow-FET). Vortex flow patterns of field-effect-tunable BICEO with two distinct gate voltage sequences are calculated numerically, with the simulation results displayed in Fig.4, wherein the amplitude of driving voltage is fixed at $2 \mathrm{~V}$. In order to induce asymmetric ICEO whirlpools, all the gate voltages deviate from the ground condition, and instead powered by the remaining two output ports of the waveform generator $(1 \mathrm{~V})$, while the exciting frequency is kept constant at $50 \mathrm{~Hz}$ (Fig.4). The correspondingly experimental results are demonstrated in Fig.5.

In Fig.4(a), the two circular gate strips $\left(2^{\text {nd }}\right.$ and $\left.4^{\text {th }}\right)$ are subjected to ac voltage signals of $\cos \left(\omega \mathrm{t}+180^{\circ}\right)$ and $\cos (\omega \mathrm{t})$, with potential phasor equivalent to $-1 \mathrm{~V}$ and $1 \mathrm{~V}$, respectively. Implementing ac signals of identical actuating frequency but different complex amplitude causes electric field intensity to be highly asymmetric along the axial direction of circular electrode array; a voltage difference of $3 \mathrm{~V}$ between the $1^{\text {st }}$ and $2^{\text {nd }}$, and $3^{\text {rd }}$ and $4^{\text {th }}$ terminals is larger than that of $1 \mathrm{~V}$ between the $2^{\text {nd }}$ and $3^{\text {rd }}$, and $4^{\text {th }}$ and $1^{\text {st }}$ ones. Since ICEO grows quadratically with imposed field strength, the strongest electroconvection appears above these gaps between the $2^{\text {nd }}$ and $3^{\text {rd }}$, and $4^{\text {th }}$ and $1^{\text {st }}$ terminals, while the flow velocity is rather weak within other interelectrode spacing. So, nanoparticles are prone to gather at the center of strong ICEO eddies, namely, the right side on the $1^{\text {st }}$ and $3^{\text {rd }}$ terminal and left side on the $2^{\text {nd }}$ and $4^{\text {th }}$ terminal (Fig.4(a) and Fig.5(b)), where the hydraulic pressure attains a local minimum value. In view of this, with field-effect flow control on nonlinear electroosmosis, the number of trapping lines of maximum fluorescence intensity doubles in the microdevice, and latex beads accumulate on all the twenty circular metal strips, in stark contrast with ten annular trapping lines formed on every other electrode bar using the typical BICEO technique (Fig.3(a)(c)). At the same time, the gap between neighboring collection lines is no longer equidistant, 
and spatial periodicity of colloid assembly pattern is characterized by repetitive alternations of one relatively small and one relatively large grating spacing in practical experiments (Fig.5(b)).

In Fig.4(b), on application of ac signals of $\cos (\omega \mathrm{t})$ and $\cos \left(\omega \mathrm{t}+180^{\circ}\right)$ to the $2^{\text {nd }}$ and $4^{\text {th }}$ terminals, respectively, since polarity of the imposed gate voltage reverses with respect to that in Fig.4(a), the ac electric field redistributes dramatically. Accordingly, both the ICEO vortex flow field (Fig.4(b)) and the circular focusing lines obtained in experiment (Fig.5(c)) are position-shifted by a quarter of the wavelength, implying a high degree of freedom controllability on the location-specific arrangement of nanoparticle trapping lines with ac-flow-FET. From above analysis and experimental test, by imposing biased ac potential waves to the two original floating terminals, we can achieve field-effect control on the flow pattern of BICEO vortex streaming, which not only greatly enhances the concentration performance, but is able to flexibly reshape the assembly pattern by regrouping the gate potential waves of opposite electrical polarities as well (see Supporting Movie 2 and 3).

\subsection{Fast self-assembly via DC-biased BICEO}

DC-biased nonlinear electrokinetics, mostly asymmetrically-polarized ACEO and ACET have become a hot topic of research over the past ten years[60]. To our best knowledge, however, scientific reports on the importance of dc-biased ac voltages in ICEO as well as its effect on tracer particles have been quite limited so far.[38] Taking this aspect into account, it is then necessary to have a check on how a hybrid de/ac voltage signal exerts an influence on the flow field of HEK, which is tightly associated with the resulted concentration performance of fluidic samples (see Supporting Movie 4).

In fact, to impose a dc voltage difference between $1^{\text {st }}$ and $3^{\text {rd }}$ terminals with one pre-existing ac electric field can make the flow behavior of electroosmosis more complicated, since this is nearly equivalent to the situation of multifrequency actuation. Under Debye-Huckel limit, however, a preliminary analysis in analogy to multifrequency DEP[56] indicates the electrostatic field under electrode polarization has a linear response to each Fourier modes of different driving frequencies. 
Accordingly, we solve the dc and ac fields separately, and then stack up the steady electroconvection of ac-ICEO, dc-ICEO and linear DCEO to obtain the effective HEK streaming, since the time-average of the cross product of counterionic charge and electric field of different oscillating frequencies is zero all the time.

In the analysis of dc component, electrochemical polarization on the driving electrodes $\left(1^{\text {st }}\right.$ and $\left.3^{\text {rd }}\right)$ cannot be fully developed with severe redox reactions on the conducting surfaces, and it is assumed that half of applied dc voltage $\mathrm{V}_{\mathrm{dc}} / 2=1 \mathrm{~V}$ would penetrate out of the boundary layer, further polarize the two floating terminals $\left(2^{\text {nd }}\right.$ and $\left.4^{\text {th }}\right)$ in direct contact with electrolyte. Besides, under dc limit, complete Debye screening occurs on the bipolar electrodes due to negligible electrochemical ion relaxation (Fig.6(a)). As a consequence, a series of ICEO micro-vortices are induced by the hybrid dc/ac voltage signal supplied from the multichannel function generator, but can only disturb the flow pattern near the floating terminals on the channel bottom surface. On the other hand, a global DCEO streaming from electrostatic force within the native double layer of positive counterions gives rise to static FSLs at the center of the $3^{\text {rd }}$ terminal surface, where there is a sharp change in the direction of the dc field lines (Fig.6(a)). In this way, fluid flows from opposite directions converge and counteract one another at the circular centerline of the $3^{\text {rd }}$ terminal, and then stream upward to form closed recirculation on a relatively large scale than local ICEO vortexes (Fig.6(b)). Simultaneously, the working fluid convects downward above the $1^{\text {st }}$ strip terminal, and then diverges to both sides. For such, in terms of achieving an equilibrium among the various vertical force components, latex beads may stay trapped by transversal electroconvection of HEK at a certain levitation height away from the flat surface of the $3^{\text {rd }}$ electrode terminal, where the downward buoyancy can balance the upward EO fluidic drag. Nevertheless, since electro-osmotic streaming cascades downward with sedimentation, it is not possible for nanoparticles to remain still above the $1^{\text {st }}$ terminal; instead, they are swept aside by the divergent HEK fluid flow on these positively-biased metal strips. Accordingly, particles can merely be captured on electrodes of negative dc polarity ( $3^{\text {rd }}$ terminal in the experiment). That is, from 
a theoretical point of view, only one annular trapping line forms in every repeating wavelength (Fig.6(b)); this is in good agreement with the five fluorescence rings collected on the annular array in practical experiment, as shown in Fig.7(b). Though the collection area with $2 \mathrm{~V}$ dc offset (Fig.7(b)) is merely half of that employing pure ac voltages (Fig.7(a)), the time required for particle preconcentration to reach the steady state decreases a lot under the synergy of linear and nonlinear EO streaming.

Since we have employed a hybrid dc/ac voltage signal in this section, nanoparticles are also subjected to DC electrophoretic (DCEP) translation in addition to viscous fluidic drag from DCEO, dc-ICEO and ac-ICEO. A superimposition of EO flow field $\boldsymbol{u}$ and DCEP motion may shift the FSL in Fig.6 and Fig.7(b) for dc-biased ac electrokinetics. However, theoretical prediction of particle collection state (Fig.6(b)) disregarding DCEP is still in good accordance with corresponding experimental observation (Fig.7(b)), implying DCEO dominates over DCEP in current situation. That is, the native zeta potential on particle surface is no more than that on insulating channel walls. In addition, it is worth mentioning that DCEP translation is opposite to DCEO streaming once both the channel wall and particle have a uniform distribution of negative free surface charge density.

\subsection{Discussion of electrothermal effect}

It is well known that electrokinetic microchips may potentially suffer from the issue of Joule medium heating across the liquid layer due to the locally amplified electric field around the microelectrode array. In our microfluidic device, the maximum temperature elevation is estimated to be negligibly small from the analytical solution[61]:

$$
\Delta \mathrm{T} \sim \quad j k \approx 0.0004 \mathrm{~K}
$$

Where $\sigma=0.001 \mathrm{~S} / \mathrm{m}$ is the electrolyte conductivity, $\mathrm{A}=2 \mathrm{~V}$ the voltage amplitude, and $\mathrm{k}=0.6 \mathrm{~W} /(\mathrm{m} \cdot \mathrm{K})$ the thermal conductivity of liquid suspension. 
Then, a scaling analysis of the ac-electrothermal (ACET) flow velocity, based upon the Stokes equation consisting of the time-averaged electrothermal body force density in dc limit, yields[62]:

$$
u_{A C E T} \sim{ }_{, L_{\mathrm{E}}}\left(\frac{1}{4} \alpha-\frac{1}{2} \beta\right) \approx 0.14 \mu \mathrm{m} / \mathrm{s}
$$

Where $L_{E}=100 \mu \mathrm{m}$ is the electrode length. $\alpha=-0.004 / \mathrm{K}$ and $\beta=0.022 / \mathrm{K}$ denote the thermal diffusivities of dielectric permittivity and electrical conductivity of saline solution, respectively. From this equation, the flow velocity of ACET is $3 \sim 4$ orders of magnitude slower than that of electroosmotic mechanism ( $100-1000 \mu \mathrm{m} / \mathrm{s})$ indicated in Eq.(6) under current experimental conditions. For this reason, the particle manipulation method presented here is completely free of electric heat generation and ACET effects, and a low temperature rise would make this technique eligible for on-chip biological applications.

\section{Concluding remarks}

In short summary, we have presented results from a mathematical analysis and experimental observation to account for electro-convective particle trapping by HEK in a four-phase spiral electrode array of five repetitive wavelength. Three distinct types of HEK having been well defined, including bi-phase ICEO, ac field-effect flow control on BICEO, and dc-biased BICEO, which can give rise to ten, twenty, five circular trapping lines of maximum fluorescence intensity in the microdevice, respectively. Besides tunable enrichment areas achievable by HEK, the location of FSL where particle trapping takes place can also be flexibly adjusted in terms of alternating the ac voltage wave along the sequentially-distributed metal strips, reversing the gate voltage polarity, and transferring the dc offset to another electrode terminal for the three forms of ICEO fluid dynamics, respectively. The numerical simulations indicate that HEK features complex rotation behaviors in hybrid dc/ac electric fields. The resultant rotational dynamics under distinct experimental conditions is shown to affect significantly the collection pattern of nanofluidic samples adjacent to ideally 
polarizable surfaces in electrolytes. These results have close bearing on several exhilarating applications, including on-chip sample detection and characterization. Flow rotation of HEK may be either favorable (stirring) or adverse (transport). The physical insights about HEK provided in current work may guide the elaborate design of high degree of freedom electrokinetic platforms to either intensify or suppress them. Further work on HEK may include the investigation of using more complex electrode structures for sample concentration and the coupling of multifrequency IDL charge with effects of a large Dukhin number, including bipolar electrochemistry, nonlinear surface capacitance, non-uniform surface conduction, ion concentration polarization, steric effect and so on. Under such situations, the linear asymptotic analysis will not work and alternative mathematical models are pursued to account for the influence of nonlinear diffuse charge dynamics on field-effect-reconfigurable nanoparticle trapping by HEK. We faithfully believe that HEK would motivate interdisciplinary research in soft matter, analytical chemistry, and electrokinetic micro/nano-fluidics in the near future.

\section{Acknowledgments}

This project is financially supported by the National Natural Science Foundation of China (No. 11672095, No. 11702035), Opening fund of State Key Laboratory of Nonlinear Mechanics, the Chang'an University Fundamental Research Funds for the Central Universities (No. 310832171008 , No. 300102328201, No. 300102328501), Shaanxi kefa [2018] No. 9 key industrial innovation chain (group)-industrial field (No. 2018ZDCXL-GY-05-04, No. 2018ZDCXL-GY-05-07-02), and Self-Planned Task (SKLRS201803B) of State Key Laboratory of Robotics and System (HIT).

The authors have declared no conflict of interest. 


\section{Nomenclature}

\section{Alphabetical symbols}

A Voltage amplitude of the applied AC signal (V)

$\mathrm{C}_{\mathrm{D}} \quad$ Diffuse layer capacitance $\left(0.0188 \mathrm{~F} \mathrm{~m}^{-2}\right) \quad C_{D}=\varepsilon / \lambda_{D}$

$\mathrm{C}_{\mathrm{S}} \quad$ Stern layer capacitance $\left(0.8 \mathrm{~F} \mathrm{~m}^{-2}\right)$

$\mathrm{C}_{0} \quad$ Linear capacity of the entire electrical double layer $\left(0.0184 \mathrm{~F} \mathrm{~m}^{-2}\right) \mathrm{C}_{0}=\mathrm{C}_{\mathrm{D}} /(1+\delta)$

D Diffusion coefficient of ionic species $\left(2 \times 10^{-9} \mathrm{~m}^{2} \mathrm{~s}^{-1}\right)$

E Electric field vector $\left(\mathrm{V} \mathrm{m}^{-1}\right)$

$\boldsymbol{E}_{\mathrm{t}} \quad$ Tangential component of electric field vector $\left(\mathrm{V} \mathrm{m}^{-1}\right)$

$E_{n}^{E D L} \quad$ Normal component of electric field with the EDL $\left(\mathrm{V} \mathrm{m}^{-1}\right)$

\section{$f \quad$ Field frequency $(\mathrm{Hz})$}

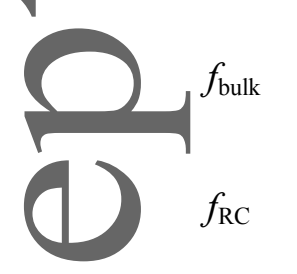

Charge relaxation frequency of fluid bulk $(225 \mathrm{KHz}) f_{\text {bulk }}=\sigma / 2 \pi \varepsilon$

$\mathrm{H} \quad$ Height of the channel $(500 \mu \mathrm{m})$

$\mathrm{h} \quad$ Levitation height from the electrode surface (m)

$\mathrm{k}$ Thermal conductivity of suspension medium $\left(0.6 \mathrm{~W} \mathrm{~m}^{-1} \mathrm{k}^{-1}\right)$

$\mathrm{L}_{\mathrm{E}} \quad$ Electrode width $(100 \mu \mathrm{m})$

$\mathrm{L}_{\mathrm{G}} \quad$ Interelectrode separation $(30 \mu \mathrm{m})$ 
L $\quad$ Signal wavelength $(520 \mu \mathrm{m})$

$\mathrm{N} \quad$ Number of AC voltage wavelength (5)

$p \quad$ Hydraulic pressure $(\mathrm{Pa})$

R Characteristic macroscopic length scale of double-layer capacitive charging (100 $\mu \mathrm{m})$

Rey The Reynolds number (0.1) Rey $=\rho_{\mathrm{f}} \mathrm{u} R / \eta$

$\mathrm{r} \quad$ Radius of latex beads $(250 \mathrm{~nm})$

$\mathrm{T} \quad$ Temperature field $(\mathrm{K})$

t Time (s)

$\boldsymbol{u} \quad$ Flow velocity vector $\left(\mathrm{m} \mathrm{s}^{-1}\right)$

$x \quad \mathrm{X}$ coordinate $(\mathrm{m})$

$y \quad$ Y coordinate $(\mathrm{m})$

Greek symbols

Liquid permittivity $\left(7.08 \times 10^{-10} \mathrm{~F} \mathrm{~m}^{-1}\right)$

Electrolyte conductivity $\left(0.001 \mathrm{~S} \mathrm{~m}^{-1}\right)$

$\sigma_{\text {free }} \quad$ Free surface charge density on insulating channel walls $\left(-0.941 \mathrm{mC} \mathrm{m}^{-2}\right) \sigma_{\text {free }}=\varepsilon \zeta_{\text {fixed }} / \lambda_{D}$

$\phi \quad$ Electrostatic potential field (V)

$\alpha \quad$ Thermal diffusivity of dielectric permittivity $\left(-0.004 \mathrm{~K}^{-1}\right) \quad \alpha=\partial \varepsilon / \partial T / \varepsilon$

$\beta \quad$ Thermal diffusivity of liquid conductivity $\left(0.022 \mathrm{~K}^{-1}\right) \quad \beta=\partial \sigma / \partial T / \sigma$ 
$\eta \quad$ Dynamic viscosity of water $\left(0.001 \mathrm{~Pa} \mathrm{~s}^{-1}\right)$

$\omega \quad$ Angular field frequency $\left(\operatorname{rad~s}^{-1}\right) \omega=2 \pi f$

$\rho_{f} \quad$ Mass density of water $\left(1000 \mathrm{~kg} \mathrm{~m}^{-3}\right)$

$\rho_{p} \quad$ Mass density of polystyrene nanospheres $\left(1050 \mathrm{~kg} \mathrm{~m}^{-3}\right)$

$\rho_{\text {free }} \quad$ Free charge density $\left(\mathrm{C} \mathrm{m}^{-3}\right) \rho_{\text {free }}=\nabla \cdot(\varepsilon \mathrm{E})$

$\tau_{\mathrm{RC}} \quad$ Double-layer charge relaxation time (s) $\tau_{R C}=R C_{D} / \sigma(1+\delta)$

$\delta \quad$ Surface capacitance ratio $(0.024) \delta=\mathrm{C}_{\mathrm{D}} / \mathrm{C}_{\mathrm{S}}$

$\zeta_{\mathrm{i}} \quad$ Induced zeta potential on ideally polarizable surfaces (V)

$\zeta_{\text {fixed }} \quad$ Fixed zeta potential across the native EDL adjacent to channel walls $(-50 \mathrm{mV})$

$\phi_{\mathrm{i}} \quad$ The body potential of $i$ th terminal (V)

$\lambda_{\mathrm{D}} \quad$ Debye screening length $(37.6 \mathrm{~nm}) \quad \lambda_{D}=\sqrt{D \varepsilon / \sigma}$

Subscripts and superscripts

ACET AC electrothermal

ac Alternating current

ac-ICEO ICEO convection induced by ac field components

bulk Fluid bulk

D Diffuse layer

DCEO Linear electroosmosis in DC electric fields. 


\section{dc Direct current}

dc-ICEO ICEO convection induced by dc field components

E Electrode

EDL Electric double layer

f Liquid suspension

free $\quad$ Free charge density

i The $i$ th terminal

n Normal component

p Particle

RC Resistor capacitor

S Stern layer

t Tangential component

Mathematical symbols

$\langle$ A $\quad$ Time-average operator

A Phasor amplitude

$\operatorname{Re}(\mathrm{A}) \quad$ Real part operator

Complex conjugate operator

\section{References}


[1] American Public Health Association, American Water Works Association, Water Pollution Control Federation, Water Environment Federation, Standard methods for the examination of water and wastewater, American Public Health Association, 1915.

[2] Visor, G. C., Schulman, S. G., Journal of pharmaceutical sciences 1981, 70, 469-475.

[3] Tanyeri, M., Schroeder, C. M., Nano letters 2013, 13, 2357-2364.

[4] Tallapragada, P., Hasabnis, N., Katuri, K., Sudarsanam, S., Joshi, K., Ramasubramanian, M., Journal of Micromechanics \& Microengineering 2015, 25, 084013.

[5] Wang, C., Sun, S., Chen, Y., Cheng, Z., Li, Y., Jia, L., Lin, P., Yang, Z., Shu, R., Microfluidics \& Nanofluidics 2018, 22, 25.

[6] Ding, X., Lin, S.-C. S., Kiraly, B., Yue, H., Li, S., Chiang, I.-K., Shi, J., Benkovic, S. J., Huang, T. J., Proceedings of the National Academy of Sciences 2012, 109, 11105-11109.

[7] Collins, D. J., Ma, Z., Ye, A., Analytical Chemistry 2016, 88, 5513.

[8] Antfolk, M., Muller, P. B., Augustsson, P., Bruus, H., Laurell, T., Lab on A Chip 2014, 14, 2791-2799.

[9] García-Arribas, A., Martínez, F., Fernández, E., Ozaeta, I., Kurlyandskaya, G. V., Svalov, A. V., Berganzo, J., Barandiaran, J. M., Sensors \& Actuators A Physical 2011, 172, 103-108.

[10] Khashan, S. A., Dagher, S., Alazzam, A., Mathew, B., Hilalalnaqbi, A., Journal of Micromechanics \& Microengineering $2017,27,055016$.

[11] Chen, Q., Li, D., Malekanfard, A., Cao, Q., Lin, J., Wang, M. H., Han, X., Xuan, X., Analytical Chemistry 2018.

[12] Ko, S. H., Song, Y.-A., Kim, S. J., Kim, M., Han, J., Kang, K. H., Lab on a Chip 2012, 12, 4472-4482.

[13] Liu, W., Ren, Y., Tao, Y., Yao, B., Liu, N., Wu, Q., Physics of Fluids 2017, 29, 112001.

[14] Dovichi, N. J., Electrophoresis 1997, 18, 2393-2399.

[15] Franze, B., Strenge, I., Engelhard, C., Journal of Analytical Atomic Spectrometry 2017, 32.

[16] Riley, K. R., Liu, S., Guo, Y., Libby, K., Cubicciotti, R., Colyer, C. L., Journal of Chromatography A 2016, 1463, 169-175.

[17] Park, S., Koklu, M., BeskoK, A., Analytical chemistry 2009, 81, 2303-2310. 
[18] Kale, A., Song, L., Lu, X., Yu, L., Hu, G., Xuan, X., Electrophoresis 2017.

[19] Ruud, E. D., Dutcher, C. S., Physical Review E 2018, 97.

[20] Chen, D., Du, H., Microfluidics and Nanofluidics 2009, 9, 281-291.

[21] Kale, A., Patel, S., Xuan, X., Micromachines 2018, 9, 123.

[22] Kale, A., Lu, X., Patel, S., Xuan, X., Journal of Micromechanics \& Microengineering 2014, 24, 75007-75012(75006).

[23] Lalonde, A., Romerocreel, M. F., Saucedoespinosa, M. A., Lapizcoencinas, B. H., Biomicrofluidics 2015, 9, 51.

[24] Harnett, C. K., Templeton, J., Dunphy-Guzman, K. A., Senousy, Y. M., Kanouff, M. P., Lab on a Chip 2008, 8, $565-572$.

[25] Jain, M., Yeung, A., Nandakumar, K., Biomicrofluidics 2009, 3, 022413.

[26] Wu, Z., Li, D., Electrochimica Acta 2008, 53, 5827-5835.

[27] Ren, Y., Liu, W., Jia, Y., Tao, Y., Shao, J., Ding, Y., Jiang, H., Lab on a chip 2015, 15, 2181-2191.

[28] Ren, Y., Liu, J., Liu, W., Lang, Q., Tao, Y., Hu, Q., Hou, L., Jiang, H., Lab on a Chip 2016, 16, 2803-2812.

[29] Tao, Y., Ren, Y., Liu, W., Wu, Y., Jia, Y., Lang, Q., Jiang, H., Electrophoresis 2016, 37, 1326-1336.

[30] Liu, W., Shao, J., Ren, Y., Liu, J., Tao, Y., Jiang, H., Ding, Y., Biomicrofluidics 2016, 10, 034105.

[31] Liu, W., Shao, J., Jia, Y., Tao, Y., Ding, Y., Jiang, H., Ren, Y., Soft matter 2015, 11, 8105-8112.

[32] Ren, Y., Liu, X., Liu, W., Tao, Y., Jia, Y., Hou, L., Li, W., Jiang, H., Electrophoresis 2018, 39, 597-607.

[33] Ramos, A., Electrokinetics and electrohydrodynamics in microsystems, Springer Science \& Business Media 2011.

[34] Schasfoort, R. B., Schlautmann, S., Hendrikse, J., van den Berg, A., Science 1999, 286, 942-945.

[35] Van Der Wouden, E., Heuser, T., Hermes, D., Oosterbroek, R., Gardeniers, J., Van Den Berg, A., Colloids and Surfaces A: Physicochemical and Engineering Aspects 2005, 267, 110-116.

[36] Van Der Wouden, E., Hermes, D., Gardeniers, J., Van Den Berg, A., Lab on a Chip 2006, 6, 1300-1305.

[37] Cahill, B. P., Heyderman, L. J., Gobrecht, J., Stemmer, A., Physical review E 2004, 70, 036305.

[38] Prabhakaran, R. A., Zhou, Y., Zhao, C., Hu, G., Song, Y., Wang, J., Yang, C., Xuan, X., Physics of Fluids 2017, 29, $42-48$.

This article is protected by copyright. All rights reserved. 
[39] Bazant, M. Z., Kilic, M. S., Storey, B. D., Ajdari, A., Advances in colloid and interface science 2009, 152, 48-88.

[40] Schnitzer, O., Yariv, E., Physical Review E 2012, 86, 061506.

[41] Bazant, M. Z., Squires, T. M., Physical Review Letters 2004, 92, 066101.

[42] Squires, T. M., Bazant, M. Z., Journal of Fluid Mechanics 2004, 509, 217-252.

[43] Studer, V., Pépin, A., Chen, Y., Ajdari, A., Analyst 2004, 129, 944-949.

[44] Ramos, A., Morgan, H., Green, N. G., González, A., Castellanos, A., Journal of Applied Physics 2005, $97,084906$.

[45] Liu, W., Shao, J., Ren, Y., Wu, Y., Wang, C., Ding, H., Jiang, H., Ding, Y., Journal of Micromechanics \& Microengineering 2016, 26, 095003.

[46] Ren, Y., Liu, W., Wang, Z., Tao, Y., Physics of Fluids 2018, 30, 062006.

[47] Pascall, A. J., Squires, T. M., Physical review letters 2010, 104, 088301.

[48] Liu, W., Ren, Y., Tao, Y., Li, Y., Chen, X., Electrophoresis 2017.

[49] Eden, A., Mccallum, C., Storey, B. D., Pennathur, S., Meinhart, C. D., Phys.rev.fluids 2017, 2.

[50] Garcíasánchez, P., Ramos, A., Langmuir the Acs Journal of Surfaces \& Colloids 2017, 33, 8553.

[51] Zhao, C. L., Yang, C., Electrophoresis 2011, 32, 629-637.

[52] Schnitzer, O., Frankel, I., Yariv, E., Journal of Fluid Mechanics 2013, 722, 394-423.

[53] Ren, Y., Liu, W., Tao, Y., Hui, M., Wu, Q., Micromachines 2018, 9, 102.

[54] Khair, A. S., Squires, T. M., Physics of Fluids (1994-present) 2008, 20, 087102.

[55] Yossifon, G., Frankel, I., Miloh, T., Physics of Fluids 2007, 19, 217.

[56] Urdaneta, M., Smela, E., Electrophoresis 2007, 28, 3145-3155.

[57] Mavrogiannis, N., Desmond, M., Gagnon, Z. R., Electrophoresis 2015, 36, 1386-1395.

[58] Mavrogiannis, N., Desmond, M., Ling, K., Fu, X., Gagnon, Z., Micromachines 2016, 7, 214.

[59] Liu, W., Ren, Y., Tao, Y., Chen, X., Yao, B., Hui, M., Bai, L., Physics of Fluids 2017, 29, 112002. 
[60] Ng, W. Y., Goh, S., Lam, Y. C., Yang, C., Rodríguez, I., Lab on a Chip 2009, 9, 802-809.

[61] Ramos, A., Morgan, H., Green, N. G., Castellanos, A., Journal of Physics D: Applied Physics 1998, 31, 2338.

[62] Liu, W., Ren, Y., Tao, Y., Yao, B., Li, Y., Electrophoresis 2018, 39, 779-793. 


\section{A list of Figures:}
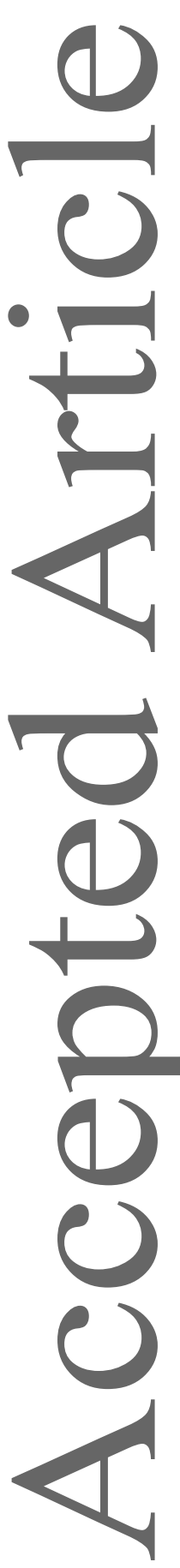

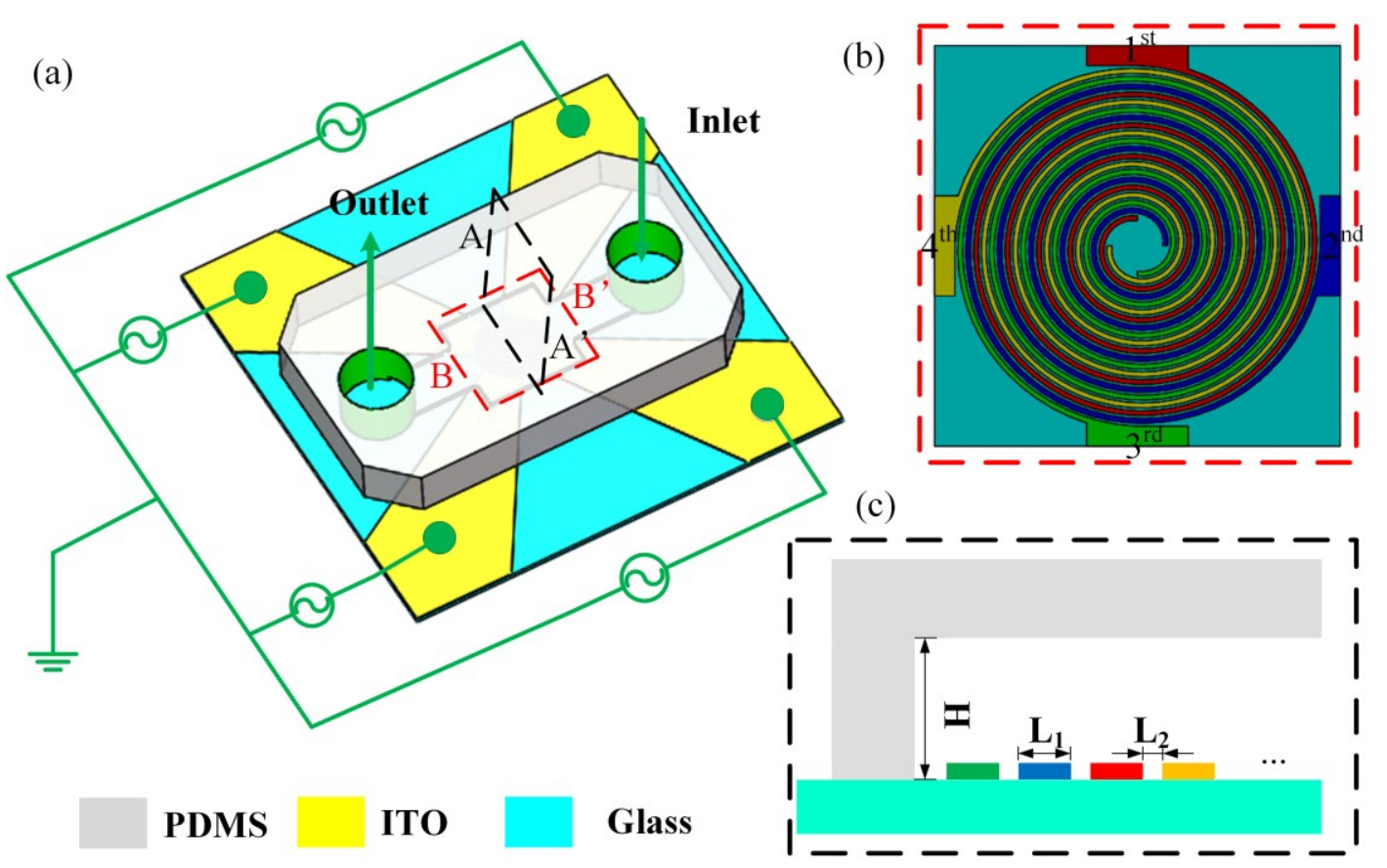

Figure 1. A 3D schematic representation of the experimental facility. (a) A confocal spiral electrode array of four ITO terminals is disposed on a glass base. There are $\mathrm{N}=5$ repeating cycles of the four-phase metal strips. Every annular conductive electrode has a span of $L_{1}=100 \mu \mathrm{m}$, with an interelectrode gap size of $\mathrm{L}_{2}=30 \mu \mathrm{m}$. A PDMS microchamber of $\mathrm{H}=500 \mu \mathrm{m}$ in height with an electrolytic port on both sides is bonded with the insulating substrate to form an intact microfluidic chip. $\mathrm{KCl}$ aqueous electrolyte containing nanofluidic samples is injected into and ultimately stuffs the microchannel. Different combinations of hybrid dc/ac voltage signals are applied to the four electrode terminals to actuate distinct vortex flow patterns of HEK on the circulating electrode array for preconcentration of nanoparticles at the flow stagnation lines on the surface of the sequential metal strips (b) 2D Geometrical configuration of the four-terminal annular electrode array in the horizontal cross-section B-B' (red dotted rectangle in (a)), with the $1^{\text {st }}, 2^{\text {nd }}, 3^{\text {rd }}$ and $4^{\text {th }}$ flat terminals colored in red, blue, green and yellow, respectively. (c) Device geometry in the transverse cross-section A-A' (black dotted rectangle in (a)). 

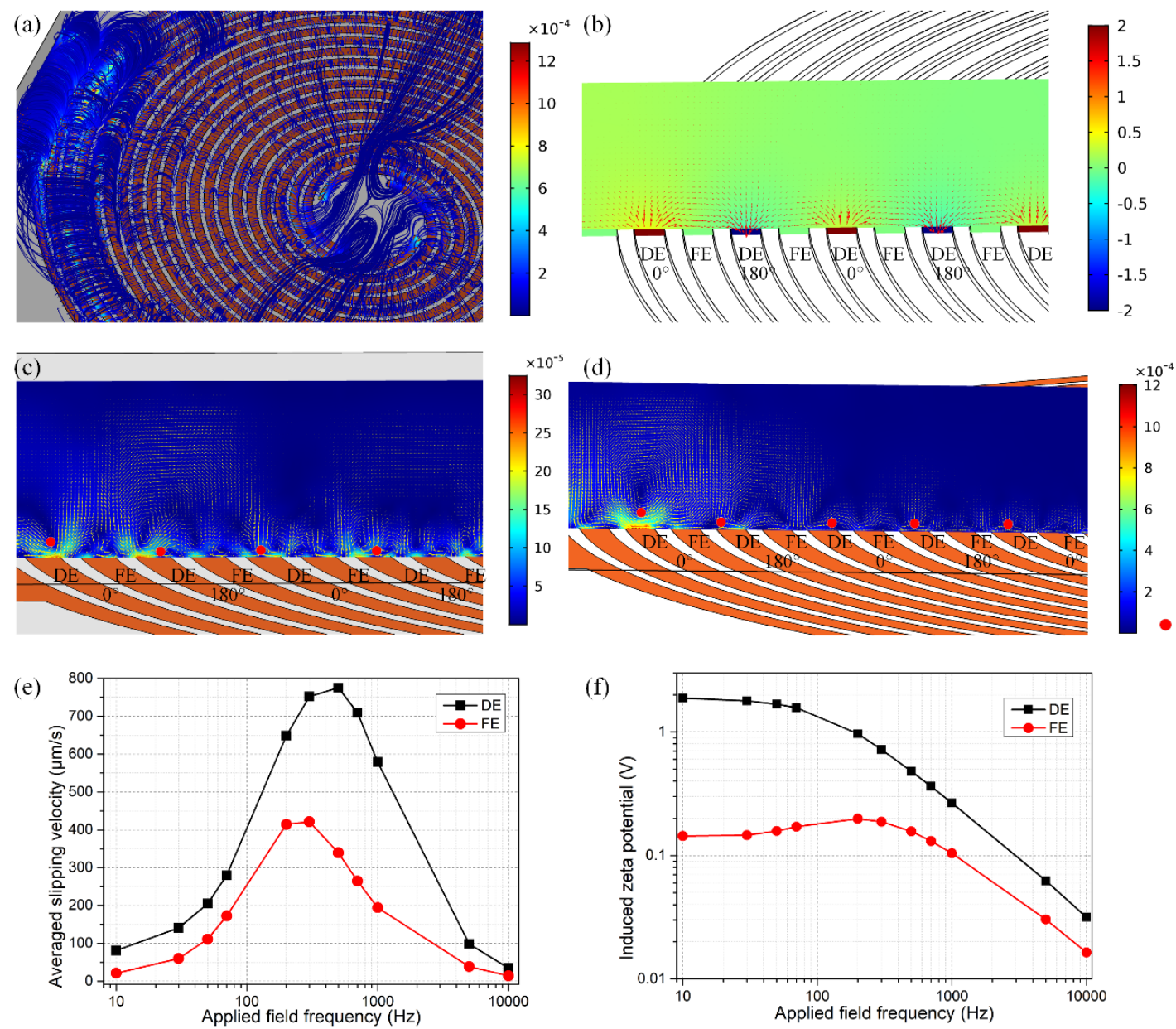

Figure 2. Theoretical analysis of particle enrichment in the four-terminal spiral electrode array via BICEO under given field frequency $f=50 \mathrm{~Hz}$ and voltage amplitude $\mathrm{A}=2 \mathrm{~V}$. (a) A global view of vortex streamlines of BICEO over the annular electrode array in the microchamber (unit: $\mathrm{m} / \mathrm{s}$ ), (b) a surface plot of potential phasor and an arrow plot of electric field vector in the cross-section A-A' for $A_{1}=2 \mathrm{~V}$ and $A_{3}=-2 V$, while $2^{\text {nd }}$ and $4^{\text {th }}$ terminals floats in the harmonic field (unit: $V$ ). (c)-(d) A cross-sectional surface and arrow plot of HEK vortex flow field (unit: $\mathrm{m} / \mathrm{s}$ ), (c) as the ac forcing is emitted from the $1^{\text {st }}$ and $3^{\text {rd }}$ terminals, i.e. the working status 1 , (d) as the $2^{\text {nd }}$ and $4^{\text {th }}$ terminals provide the necessary field source and sink, i.e. the working status 2 , it is worthy to note that the nanoparticle samples tend to always collect on the ten driving electrodes rather than the two floating terminals, so we are able to realize an in-time switching of particle collection positions (the red solid spheres) by alternating the voltage supply modes between state 1 and 2 with suitable time intervals. (e) A comparison between the frequency-dependent BICEO flow velocity on DEs and FEs, where there is a single relaxation 
peak for both ACEO and ICEO vortex streaming. (f) Real part of phasor amplitude of the induced zeta potential across the EDL on both DE and FE as a function of applied field frequency.

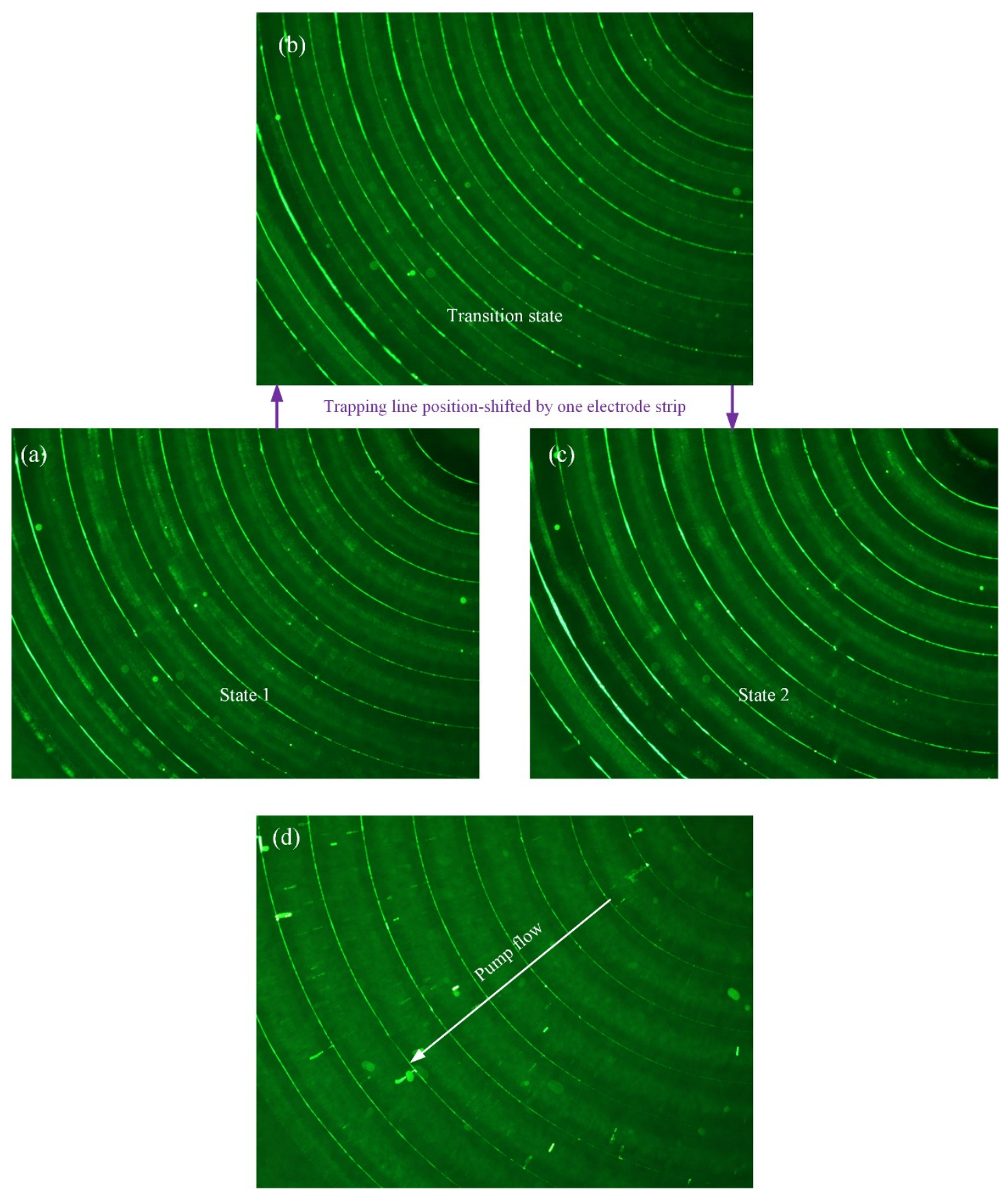

Figure 3. Experimental observation of nanoparticle enrichment using BICEO with a concentration ratio of 36.3. (a) A microscopic view of particle collection pattern for status 1 with the $1^{\text {st }}$ and $3^{\text {rd }}$ terminals electrically activated, (b) experimental result of an intermediate transition status between 
state 1 and 2, (c) configuration of particle self-assembly line for status 2 with the $2^{\text {nd }}$ and $4^{\text {th }}$ terminals powered on, (d) an outward pumping flow component caused by bi-phase electroosmotic convection above the annular strip array, enhancing the particle trapping performance to some degree. (See Supporting Movie 1) 

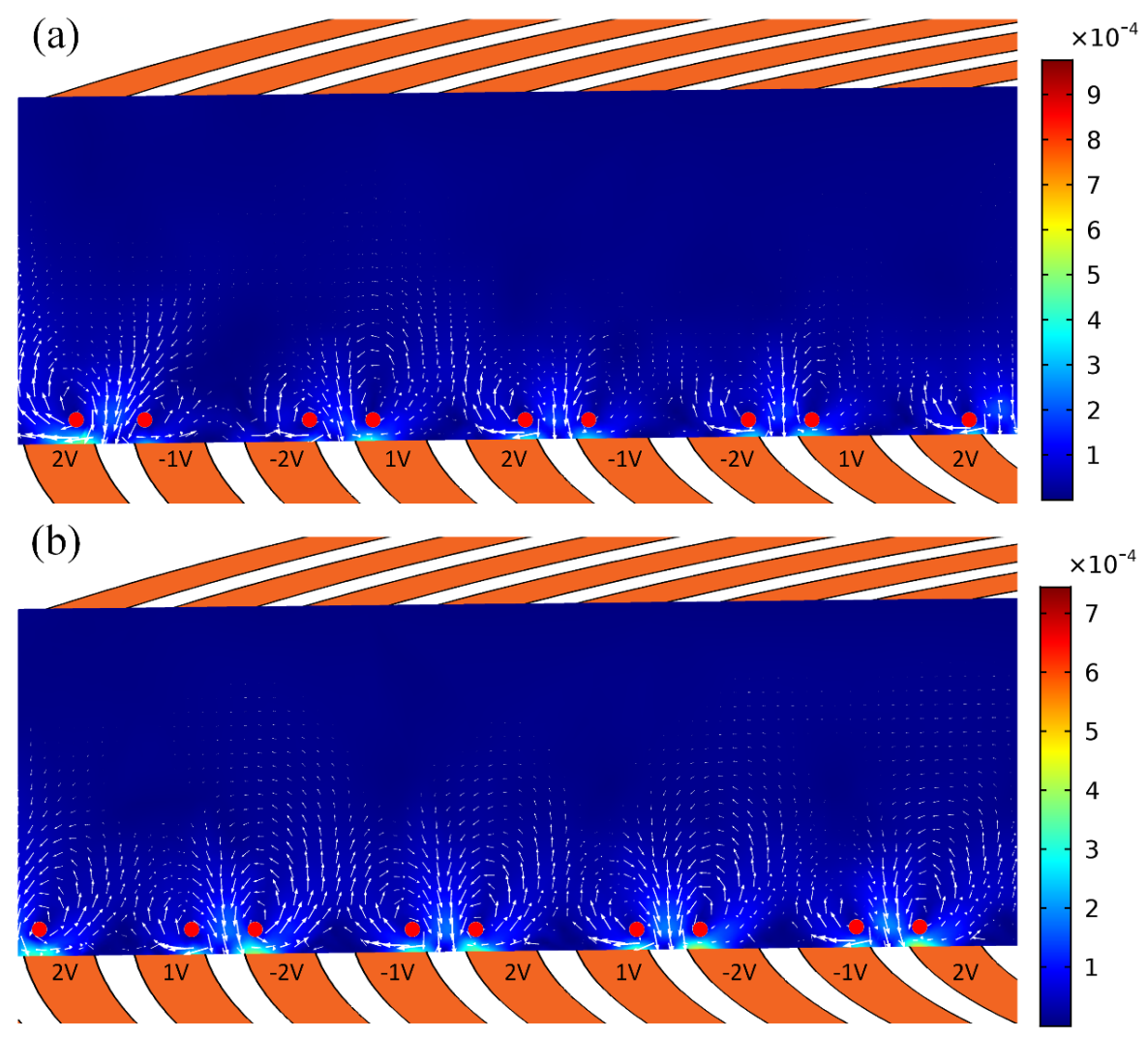

Figure 4. Theoretical prediction of vortex flow field of ac-flow-FET of BICEO and the resulting collection state of nanofluidic samples, with the $1^{\text {st }}$ and $3^{\text {rd }}$ terminals subjected to driving voltages $2 \cos (\omega \mathrm{t})[\mathrm{V}]$ and $2 \cos \left(\omega \mathrm{t}+180^{\circ}\right)[\mathrm{V}]$, respectively, while two distinct combination of gate voltages are imposed to the $2^{\text {nd }}$ and $4^{\text {th }}$ terminals. (a) A surface and arrow plot of asymmetric vortex streaming of BICEO flow-FET for $\mathrm{V}_{2}=\cos \left(\omega \mathrm{t}+180^{\circ}\right)$ and $\mathrm{V}_{4}=\cos (\omega \mathrm{t})$, (b) once the gate polarities make a reversal, viz. $\mathrm{V}_{2}=\cos (\omega \mathrm{t})$ and $\mathrm{V}_{4}=\cos \left(\omega \mathrm{t}+180^{\circ}\right)$, the circular trapping lines are position-shifted by a quarter of the wavelength. The red spheres indicate that, it is highly possible for nanoparticles to amass in the vicinity of potent flow regions of asymmetric ICEO micro-vortices along the electrode array. 

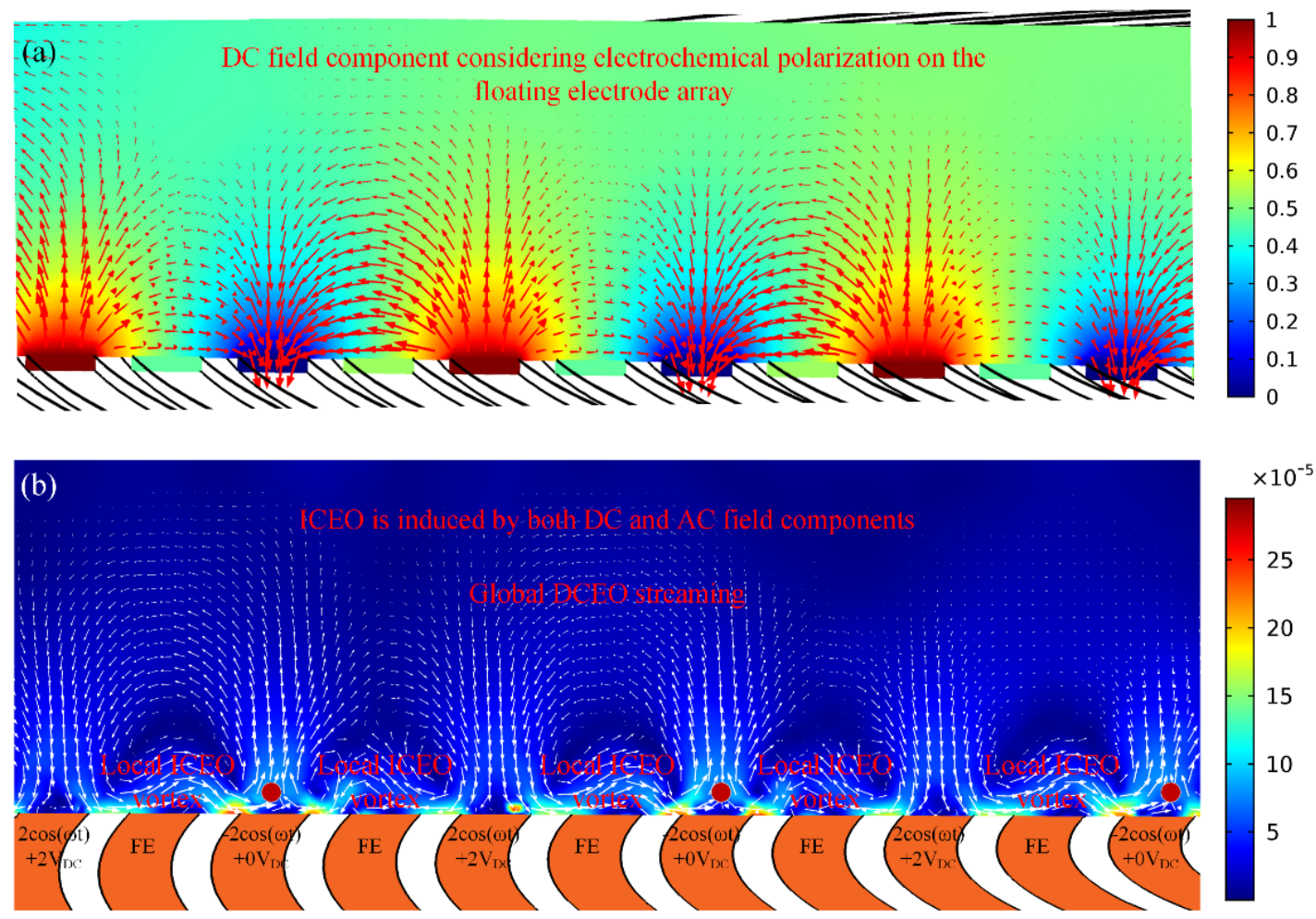

Figure 6. Theoretical prediction on the effect of dc-biased ac-field induced-charge electroosmosis on enrichment of nanoparticles in the annular electrode array. (a) A surface and arrow plot of dc field component considering electrode polarization (unit: V). (b) A surface and arrow plot of the flow field of HEK on application of a hybrid ac/dc voltage difference between the $1^{\text {st }}$ and $3^{\text {rd }}$ terminals, with other bipolar circular strips acting as floating electrodes (unit: $\mathrm{m} / \mathrm{s}$ ). Nanoparticles (red spheres) have a propensity to collect above the $3^{\text {rd }}$ electrode terminal in every repeating wavelength. 

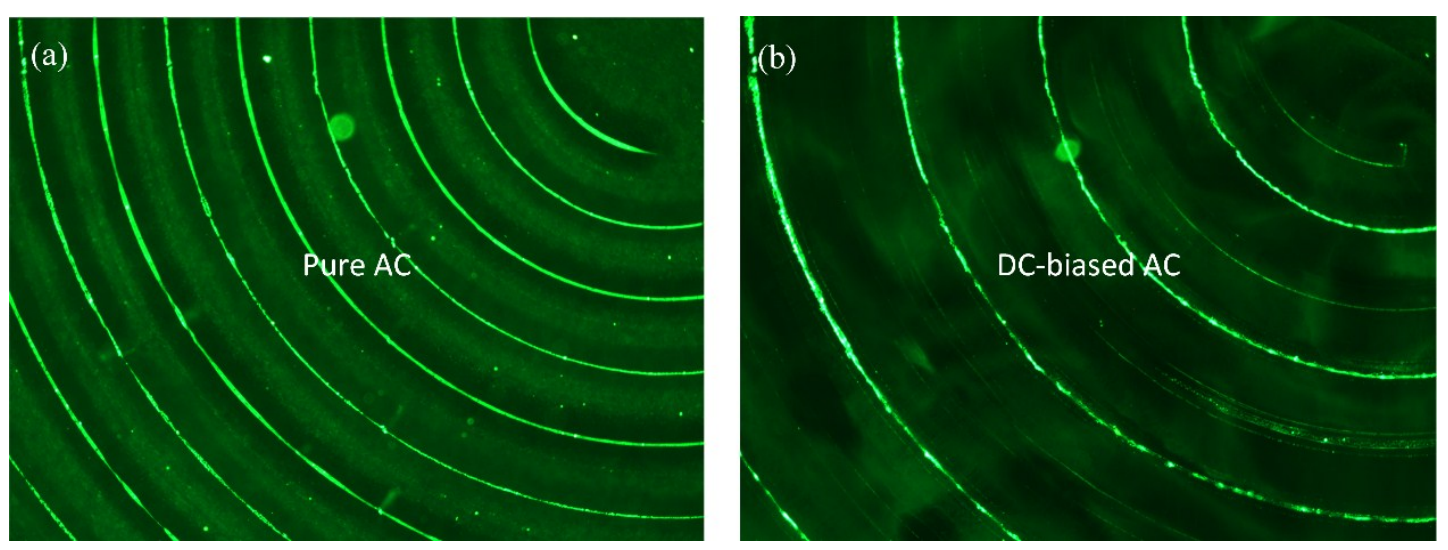

Figure 7. A comparison between particle trapping experiment with and without a dc voltage offset. (a) In sheer ac fields, ten particle trapping lines are formed above the two spiral driving terminals; (b) Under an additional dc bias, though the area of enrichment is reduced by half, the collection process becomes much faster under the simultaneous action of linear and nonlinear electro-convective streaming with a concentration ratio of 58.8. (See Supporting Movie 4) 


\section{A list of Tables:}

Table 1. Boundary conditions for current conservation in ac and dc fields

\begin{tabular}{|c|c|c|c|}
\hline Index & Boundary & $\begin{array}{c}\text { Boundary conditions for ac } \\
\text { field component Eq.(A1) }\end{array}$ & $\begin{array}{c}\text { Boundary conditions for dc } \\
\text { field component Eq.(A2) }\end{array}$ \\
\hline 1 & $1^{\text {st }}$ terminal & $\sigma \boldsymbol{n} \cdot \nabla \phi_{\mathrm{ac}}=j \omega \frac{C_{\mathrm{D}}}{1+\delta}\left(\phi_{\mathrm{ac}}-\phi_{\mathrm{acl}}\right)$ & $\phi_{d c 1}=1 \mathrm{~V}$ \\
\hline 2 & $2^{\text {nd }}$ terminal & $\sigma \boldsymbol{n} \cdot \nabla \phi_{\mathrm{ac}}=j \omega \frac{C_{\mathrm{D}}}{1+\delta}\left(\phi_{\mathrm{ac}}-\phi_{\mathrm{ac} 2}\right)$ & $\sigma \boldsymbol{n} \cdot \nabla \phi_{d c}=0, \phi_{d c 2}=0.5 \mathrm{~V}$ \\
\hline 3 & $3^{\text {rd }}$ terminal & $\sigma \boldsymbol{n} \cdot \nabla \phi_{\mathrm{ac}}=j \omega \frac{C_{\mathrm{D}}}{1+\delta}\left(\phi-\phi_{\mathrm{ac} 3}\right)$ & $\phi_{\mathrm{dc} 3}=0 \mathrm{~V}$ \\
\hline 4 & $4^{\text {th }}$ terminal & $\sigma \boldsymbol{n} \cdot \nabla \phi_{\mathrm{ac}}=j \omega \frac{C_{\mathrm{D}}}{1+\delta}\left(\phi-\phi_{\mathrm{ac} 4}\right)$ & $\sigma \boldsymbol{n} \cdot \nabla \phi_{d c}=0, \phi_{d c 4}=0.5 \mathrm{~V}$ \\
\hline 5 & Insulating surfaces & $\boldsymbol{n} \cdot \nabla \phi_{\mathrm{ac}}=0$ & $\boldsymbol{n} \cdot \nabla \phi_{d c}=0$ \\
\hline
\end{tabular}

